\title{
INTENSIFIED ELECTRON-BOMBARDED CCD IMAGES FOR INDUSTRIAL AND SCIENTIFIC APPLICATIONS
}

\author{
I. Dalinenko, G. Vishnevsky, V. Kossov, L. Lasovsky, G. Kuzmin and A. Malyarov \\ "Electron" Research Institute, St. Petersburg
}

\section{INTRODUCTION}

Electron-Bombarded CCD (EB-CCD) image intensifiers, based on the primary photoelectron multiplication effect in the CCD substrate, provide a number of advantages over routine intensified CCDs, optically coupled with the output of conventional image intensifiers. Despite this fact, there are no commercially available intensified EB-CCD imagers, though many CCD and image intensifier's manufactures put great efforts to design devices of such kind. This paper briefly reviews the results of more than a decade of work in the field of intensified EB-CCD imaging devices in the "Electron" National Research Institute, Russia's leader in optoelectronics.

\section{THE DESIGN AND MANUFACTURE OF THE EB-CCD IMAGING TUBES IN RUSSIA - STATE OF THE ART}

\subsection{ICCD-16 - the Basic Model of the EB-CCD Imaging Tubes}

As the result of prolonged efforts with the semi-industrial technology of the intensified EB-CCD, an image tube (ICCD-16) has been created with 520 × 580 pixels. This device can operate in the faceplate illuminance range from 50 down to $10^{-4} \mathrm{~lx}$, providing spatial resolution not worse than 350 TV lines. When coupled with the output of Gen 1 image intensifier, this EB-CCD imaging tube provides spatial resolution not worse than 350 TV lines in the faceplate illuminance range down to $5 \times 10^{-6} \mathrm{~lx}$, permitting such "coupled" device successfully to compete with Gen 2 MCP-based intensified CCDs.

Along with the improved fabrication technique, the 780 × 580 CCD version of the initial device model has been designed, produced and tested. This EB-CCD imaging tube provides ultimate spatial resolution not worse than $600 \mathrm{TV}$ lines in the faceplate illuminance range down to $5 \times 10^{-4} \mathrm{~lx}$ and outperforms the MCP image intensifier because it allows a signal-to-noise level comparable to MCP tubes but without the MTF degradation on the high spatial frequencies.

\section{THE DEVELOPMENT OF THE EB-CCD FAMILY IN THE NEAR FUTURE}

The totality of experience in design and manufacturing of the EB-CCD Imaging Tubes permits us to proceed in this work in the direction of creating new devices of this family, which could widen the variety of applications. The design process of the devices proposed has already begun and some of them are scheduled to be manufactured in the near future. 


\subsection{Intensified EB-CCD Imaging Device With a 1024 x 1024 Full-Frame CCD.}

This device is based on the scientific-oriented image-converter tube "Yasen" and is intended for the slow-scan or pulse mode of operation in scientific fields of applications, such as high-energy physics, astronomy, high-speed photonics etc. Demagnification of the input image size and the photoelectrons energy increase up to $15 \mathrm{keV}$ together with the low-noise CCD readout mode provide the photon counting mode with the signal-to-noise ratio not worse than five and spatial resolution not worse than $40 \mathrm{lp} / \mathrm{mm}$. The EB-CCD used is a full-frame, 1024 x 1024 pixels, $13 \mu \mathrm{m}$ x $13 \mu \mathrm{m}$, device with two (low-noise and high-speed) output amplifiers.

3.2. Intensified EB-CCD Imaging Device With Input Photo-Cathode Diameter $40 \mathrm{~mm}$ and With Input Image Demagnification

The proposed device is intended to employ a frame-transfer CCD and, thus, could operate in the standard TV mode. It will be designed on the base of the scientific-oriented image-converter tube PM-031, designed and produced by the Research Institute of Opto-Physical Measurements (Moscow) Demagnification of the input image size together with the photoelectron energy increase provide the threshold sensitivity of the device as low as $5 \mathrm{x}$ $10^{-7} \mathrm{~lx}$ and ultimate resolution $600 \mathrm{TV}$ lines, thus making this device competitive with the Gen2 image intensifiers with a cooled CCD. The device is intended for the night-vision TV systems, day-and-night TV cameras as well as for the wide variety of industry and scientific applications.

3.3 Electromagnetically-Focused Intensified CCD Imaging Device With Solar-Blind Photo-Cathode For UV And EUV Imaging.

The device is intended for the detection of the low-intensity light sources in the UV and EUV spectral ranges. This device is designed with the use of the basic ICCD-16 model construction, except for some specific differences.

With the CsI or CsTe photocathodes, this device provides a threshold sensitivity of about $5 \times 10^{-16}-5 \times 10^{-17} \mathrm{~W} /$ pixel and Solar blindness factor $>10^{-10}$. With availability of appropriate financing, all these new devices could be designed and manufactured in approximately a year.

\section{CONCLUSIONS}

As the result of prolonged efforts in the field of producing Intensified Electron-Bombarded CCD Imaging Tubes, a whole family of such devices was created for a wide range of different industrial and scientific applications. Along with the improving of the EB-CCDs fabrication techniques, some novel devices could be designed and manufactured in the near future. 